\title{
Lenguaje y elección de teorías: contra la historia oficial ${ }^{*}$
}

\author{
Language and Theory Choice: Against the Official History
}

\author{
Ricardo J. Gómez
}

\begin{abstract}
Resumen
La historia oficial del neopositivismo, en particular respecto de las propuestas de R. Carnap acerca de la aceptación-rechazo de teorías, sostiene que "Carnap [pretendió] evitar (o evadir) la idea [...] de que en la actividad de seleccionar teorías se presuponen valores [...] quería reducir la elección de teorías a un algoritmo" (Putnam 2002, p. 141). Dicha historia es la que usualmente aparece en los libros de texto sobre el positivismo y/o sobre la historia de la filosofía de las ciencias, así como en aproximaciones críticas al empirismo en general y a Carnap en particular. En el presente trabajo argumentaremos que Carnap explícitamente no llevó a cabo tal reducción y no pretendió evadir la presencia de juicios de valor, aunque de un tipo muy especial.
\end{abstract}

Palabras clave: Carnap - neopositivismo - algoritmo - juicios de valor

\begin{abstract}
The official history of neopositivism, in particular with respect to R. Carnap's proposals about the acceptance-rejection of theories, holds that "Carnap [intended] to avoid (or evade) the idea $[\ldots]$ that values $[. .$.$] are presuposed in the activity of selecting scientific$ theories [...] Carnap wanted to reduce theory choice to an algorithm" (Putnam 2002, p. 141). Such a history usually appears in textbooks on positivism and/or on history of the philosophy of science as well as in critical approaches to empirism in general and to Carnap in particular. In the present paper, we will argue that Carnap explicitly did not carry out such a reduction and did not intend to avoid the presence of value judgements, although of a very special kind.
\end{abstract}

Keywords: Carnap - neopositivism - algorithm - value judgements

\footnotetext{
* Recibido: 7 Enero 2010. Aceptado: 1 Febrero 2010.

¥California State University, Los Angeles. Para contactar al autor, por favor escriba a: rgomez@calstatela.edu. Metatheoria 1(1) (2010): 31-41. ISSN 1853-2322.

(c) Editorial de la Universidad Nacional de Tres de Febrero. Publicado en la República Argentina.
} 


\section{Introducción}

La historia oficial es acerca del neopositivismo, muy especialmente sobre las propuestas de R. Carnap acerca de la aceptación-rechazo de teorías. Dicha historia es la que usualmente aparece en los libros de texto sobre el positivismo y/o sobre la historia de la filosofía de las ciencias. Pero tal historia oficial es la aceptada explícitamente en aproximaciones críticas al empirismo en general y a Carnap en particular en autores como H. Putnam, cuando afirman que Carnap buscó "reducir la selección de hipótesis a un algoritmo" (Putnam 2002, p. 30) o, más explícitamente, cuando proponen que "Carnap [pretendió] evitar (o evadir) la idea $[\ldots]$ de que en la actividad de seleccionar teorías se presuponen valores [...] quería reducir la elección de teorías a un algoritmo" (Putnam 2002, p. 141).

Argumentaremos que Carnap explícitamente no llevó a cabo tal reducción y no pretendió evadir la presencia de juicios de valor, aunque de un tipo muy especial. Nada mejor pues que dejar que Carnap nos cuente su propia versión, parte de la cual puede ser considerada el componente fundamental de las versiones más sensatas y rigurosas de la historia oficial. Más claramente, sostendremos que dichas versiones que respetan parte importante de la propia concepción de Carnap, nos dicen la verdad, pero no toda la verdad y solamente la verdad, de ahí que resulten distorsionadoras.

\section{Carnap y la historia oficial}

Los ingredientes claves de la propia versión de Carnap acerca de cómo los científicos seleccionan hipótesis y teorías pueden resumirse como sigue:

- Dicha aceptación-rechazo se lleva a cabo a posteriori del testeo empírico, el cual ha de permitir establecer la verdad de ciertos enunciados de observación. El conjunto de dichos enunciados constituye la evidencia empírica $e$ de la hipótesis $h$ o teoría $t$ en cuestión.

- Como dicha evidencia jamás da un soporte total a la hipótesis o teoría en cuestión, el argumento cuya conclusión es $h$ o $t$ y cuyas premisas son los enunciados de observación que apoyan a $h$ o $t$ es un argumento inductivo, en tanto Carnap llama argumento inductivo a aquel en el que las premisas apoyan parcialmente a la conclusión. Además todo argumento inductivo es "en términos de probabilidad" (Carnap 1962, p. v), de ahí que "la teoría de los principios del razonamiento inductivo es la misma que la lógica de la probabilidad" (Carnap 1962, p. v).

- El concepto de probabilidad sobre el cual se basa la lógica inductiva es una relación lógica entre dos enunciados, es "el grado de confirmación de una hipótesis (o conclusión) sobre la base de cierta evidencia (o premisas)" (Carnap 1962, p. v). Carnap está aquí haciendo uso de su célebre distinción entre dos conceptos de probabilidad, Prob 1 y Prob 2. Carnap afirma que el 
de Probabilidad 1 es un concepto lógico, semántico. Una sentencia acerca de tal concepto está basada no en la observación de los hechos sino en el análisis lógico; si es verdadera, es L-verdadera (analítica) (Carnap 1962, p. 19). Probabilidad 2, a su vez, "es la frecuencia relativa (a largo plazo) de una propiedad de eventos o cosas con respecto a otra [propiedad de eventos o cosas]. Una sentencia acerca de este concepto es factual, empírica" (Carnap 1962, p. 19).

- Como consecuencia de todo lo anterior, el concepto de confirmación es "semántico, basado en el significado, y lógico, o sea, independiente de los hechos. Por lo tanto, pertenece a la lógica inductiva" (Carnap 1962, p. 19).

- Carnap distingue entre tres conceptos semánticos de confirmación: (i) el concepto clasificatorio (la hipótesis $h$ es confirmada por la evidencia e), (ii) el concepto comparativo ( $h$ es confirmada por $e$ al menos tanto como $h$ ' por $\left.e^{\prime}\right)$, y (iii) el concepto cuantitativo de confirmación ( $h$ es confirmada por $e$ en el grado $q$, 'c $(\mathrm{h}, \mathrm{e})=\mathrm{q}$ ').

- Carnap desarrolla un sistema de lógica inductiva que pretende establecer, dada una hipótesis o teoría y su correspondiente evidencia empírica, el grado de confirmación c. Para ello propuso ciertos axiomas de los cuales se derivan los principales teoremas del cálculo de probabilidades (por ejemplo, el teorema de Bayes). Por ello es que Carnap sostiene que el desarrollo de tal lógica inductiva "ayudará a clarificar los fundamentos de la inducción y, por ende, de todo el método científico".

- Ello es consistente con la reiterada afirmación en las versiones de la historia oficial de que el método científico, según Carnap, en el contexto de la justificación de hipótesis y teorías, es el método inductivo, que ha sido sistematizado formalmente en la lógica inductiva que permite establecer el grado de confirmación de una hipótesis o teoría en base a su evidencia empírica o, lo que es lo mismo, establecer la probabilidad lógica de que esa hipótesis sea verdadera en base a dicha evidencia.

- Además, todo ello parece darle la razón a Putnam cuando afirma que la selección de teorías se reduce a un algoritmo (por lo que así se evita la presencia de valores). Sin embargo, Carnap mismo nos ha de invitar a ser muy cautos al respecto.

\section{Carnap más allá de la historia oficial}

Carnap nos informa que "debe admitirse que la elección del científico de una hipótesis adecuada para la explicación de hechos observados está determinada por factores de distinto tipo. Sin embargo, la lógica inductiva tiene la tarea de representar solamente los factores lógicos y no aquellos de naturaleza práctica o metodológica" (Carnap 1962, p. 161). O sea que (1) en la elección de hipótesis, si bien la lógica inductiva (los ingredientes subsumibles en un algoritmo) son 
una condición necesaria para una aceptable selección, no son condición suficiente, (2) ello es así porque en dicha elección intervienen factores prácticos (a los que Carnap en otro contexto llama "elementos volicionales") no totalmente elucidables algorítmicamente, (3) recurrir a dichos ingredientes no conduce a la irracionalidad de las decisiones; todo lo contrario, dichos factores son elementos constitutivos del método para decidir racionalmente la elección de hipótesis o teorías.

Todo ello es fortísimo y aparentemente antiortodoxo. Cabe hacerse diversas preguntas: ¿acaso la presencia de dichos factores prácticos no introduce la presencia de ingredientes no objetivos, intereses subjetivos o grupales?, ila introducción de elementos volicionales no implica para Carnap la introducción de valores y, teniendo en cuenta la historia oficial acerca de la concepción de los juicios de valor de los neopositivistas (como juicios subjetivos), no implicaría ello la intromisión de ingredientes no-científicos, no-objetivos en la actividad científica que conduce a la aceptación-rechazo justificado de hipótesis o teorías?

La obra de Carnap nos permite reconstruir una respuesta sensata, no oficial, a cada una de dichas preguntas.

Cabe aclarar que, según Carnap, los restantes factores son no cuantificables porque "algunos de ellos [dichos factores] no pueden ser evaluados numéricamente" (Carnap 1962, p. 219), por lo que Carnap afirma, ahora más claramente que nunca, que "la lógica inductiva sola no determina ni puede determinar la mejor hipótesis dada una evidencia determinada" (Carnap 1962, p. 221). Por si esto fuera poco, Carnap agrega que tal "preferencia [por la mejor hipótesis] está determinada por factores de muy distinto tipo, entre ellos lógicos, metodológicos y factores puramente subjetivos" (Carnap 1962, p. 221). Aquí la referencia a valores parece obvia, así como parecería darse una respuesta afirmativa a nuestras dos preguntas anteriores, pues se reconoce explícitamente la presencia de ingredientes valorativos subjetivos. También cabe agregar que Carnap evita al máximo de lo posible el uso del término 'valores', aunque es obvio que están allí por su continuo uso del término 'preferencia', especialmente con connotaciones de teoría económica. Coherentemente con ello "resulta claro que la lógica inductiva sola no puede servir como guía para la vida" (Carnap 1962, p. 241).

Pero entonces, ipor qué tal intromisión de ingredientes valorativos subjetivos no medra contra la objetividad del procedimiento científico en el contexto de justificación? Responderemos ahora parcialmente a dicha pregunta, ayudándonos con la propia visión de Carnap del problema: "La distinción entre el sistema de lógica inductiva pura y los procedimientos y reglas de su aplicación para decisiones prácticas es enfatizada por la siguiente razón: el análisis de la aplicación involucra [...] en adición a consideraciones de la metodología general de la inducción ciertas consideraciones de naturaleza psicológica (por ejemplo concernientes a la medición de preferencia y valuación)" (Carnap 1962, p. 254). O sea, hay presencia de valuaciones, pero dichos juicios de valor no llevan al subjetivismo dentro de la actividad científica porque dichos juicios son 
elucidables por una disciplina científica en particular. No en vano Carnap aclara que "los problemas y dificultades aquí involucrados pertenecen a la metodología de una rama especial de la ciencia empírica, la psicología de las valuaciones como parte de la teoría de la conducta humana" (Carnap 1962, p. 254).

Carnap incluso propuso algunas reglas (cuatro en total) para la decisión práctica, la última de las cuales recomienda que "entre las posibles acciones elíjase aquella para la cual la estimación de la utilidad resultante es máxima”. Y tal utilidad no es un concepto de orden moral, pues mide la satisfacción que obtiene el sujeto. Carnap reconoce que la regla de máxima utilidad presupone que esta puede ser medida y que hay una ley cuantitativa estableciendo la utilidad como una función de la ganancia (en economía, tal ley es la ley de Bernoulli de la utilidad marginal). Pero Carnap mismo reconoce que esta ley no "puede tener hoy la forma simple establecida por Daniel Bernoulli, sino que debe tener una forma más general y complicada. Este es un problema a ser resuelto por la investigación psicológica" (Carnap 1962, p. 279).

Otra vez es obvio que Carnap reconoce la presencia de ingredientes extralógicos que involucran preferencias, pero no abandona su expectativa de que ellos sean totalmente elucidables científicamente. No hay, en última instancia, presencia de valores no analizables científicamente. La ética queda, como quedó siempre en el empirismo, fuera de la actividad científica.

En el volumen de Schilpp dedicado a Carnap, en las respuestas que este da a sus críticos, es todavía más explícito y preciso al respecto (Schilpp 1963). Allí distingue las reglas de lógica inductiva (aquellas que permiten establecer el grado de confirmación de una hipótesis o teoría de acuerdo con la evidencia empírica) de las reglas o procedimientos para aceptar-rechazar hipótesis o teorías y claramente afirma que la identificación o reducción de unas a otras debe ser rechazada: "De acuerdo con una versión ampliamente aceptada, el objetivo propio de la lógica inductiva es proveer 'reglas de aceptación' que determinan, dadas $e$ y $h$, que o $h$ ha de ser aceptada o que $h$ ha de ser rechazada, o que ha de ser dejado en suspenso. Yo no estoy de acuerdo con esta posición" (Schilpp 1963, p. 972). No extraña pues que agregue que "las reglas [de aceptación] no son reglas de lógica inductiva, puesto que involucran factores no lógicos" (Carnap 1962, p. 973).

Más importante aún es que en dichas respuestas Carnap esclarece que las valuaciones involucradas en la aceptación de hipótesis-teorías son en última instancia enunciados factuales (evitando así artificialmente el reconocimiento de la presencia de juicios de valor subjetivos y con ello toda forma de peligroso subjetivismo). Así, afirma que entre dichos enunciados conectados con valores o valuaciones se hallan los siguientes: "(a) enunciados psicológicos, sociológicos e históricos acerca de las reacciones valorativas de una persona o grupo: por ejemplo enunciados de aprobación, desaprobación o preferencia de ciertas acciones, (b) enunciados de relaciones medios-fines, por ejemplo: 'la acción de $a$ es un medio para alcanzar el objetivo b', (c) enunciados acerca de la utilidad de un posible evento para una persona determinada" (Carnap 1962, p. 999). 
Carnap evidentemente rechaza todas aquellas concepciones que consideran el conocimiento de valores como un conocimiento sui generis, esencialmente diferente del conocimiento factual o lógico.

En resumen y hasta aquí: la aceptación-rechazo de hipótesis va más allá de la elucidación por algoritmos lógico-formales, pues requiere siempre decisiones prácticas que involucran valores. Tales valores son tratables factualmente, por lo que la presencia de valores en la actividad científica no requiere del uso de juicios de valor sui generis. Esto, obviamente, ya va más allá de la historia oficial. Es la peculiar y discutible concepción positivista de los juicios de valor (como dicotómicamente distintos de los juicios de hecho) lo que le impidió a Carnap reconocer que el científico qua científico hace juicios de valor. El científico hace valuaciones, incluso en el contexto de justificación, pero todas ellas son abordables factualmente. No se requiere de la presencia de juicios de valor diametralmente distintos de los juicios científico-factuales, además de la lógica y de buena investigación empírica (psicológica-sociológica), para establecer la aceptación-rechazo de hipótesis o teorías.

Antes de ser más claros acerca de los tipos de juicios de valor que intervienen en la actividad científica de aceptación-rechazo de teorías en la versión carnapiana final, conviene referirnos a una mentada lectura de la postura de Carnap en su Logical Foundations of Probability (1962).

\section{El científico qua científico hace juicios de valor}

Ello es así, según Richard Rudner (1953), porque el científico, en tanto tal, acepta o rechaza hipótesis y, como ninguna hipótesis científica está alguna vez completamente verificada, el científico debe tomar la decisión de si la evidencia disponible es lo suficientemente fuerte para garantizar la aceptación de la hipótesis.

Además, tal decisión acerca de si la evidencia es lo suficientemente elevada ha de ser "función de la importancia, en el sentido ético típico, de cometer un error al aceptar o rechazar la hipótesis" (Rudner 1953, p. 3). Por ejemplo, si la hipótesis considerada fuera acerca de la presencia en escasa cantidad de ingredientes tóxicos en una droga, se requeriría un elevado grado de confirmación antes de aceptarla, debido a las consecuencias de cometer un error, de acuerdo con nuestros estándares morales (por el enorme valor asignado a la preservación de la vida humana). Si, por el contrario, la hipótesis es acerca de la seguridad de ciertas hebillas para ajustar cinturones, el grado de confirmación que se requeriría no sería relativamente tan alto.

La presencia de una dimensión moral es evidente: el riesgo que se desea correr en caso de cometer un error al aceptar o rechazar una hipótesis depende de cuan seriamente, en el sentido ético, se visualizan las consecuencias de cometer tal error. Y en la que parece una clara alusión a Carnap, Rudner reconoce que "[a]hora muchos empiristas que reflexionan acerca de las consideraciones anteriores, están de acuerdo en que la aceptación o el rechazo de hipótesis involu- 
cra juicios de valor, pero son reacios ha aceptar la conclusión [de que el científico qua científico hace juicios de valor]" (Rudner 1953, p. 4).

Por supuesto, la gran diferencia es que si bien en ambos casos, Carnap y Rudner, hay reconocimiento de la presencia de valores en la aceptación-rechazo de hipótesis o teorías, Rudner reconoce en ello la presencia de una inalienable dimensión ética en la actividad del científico qua científico, cosa que Carnap negó siempre en aras de preservar la objetividad de la actividad científica.

Sin embargo, Rudner cree que, justamente para preservar tal objetividad, lo que el científico no debe hacer es "cerrar los ojos al hecho de que el método científico requiere intrínsicamente tomar decisiones en términos de valores" (Rudner 1953, p. 6). Se mantiene pues el ideal de objetividad, entendida como requiriendo al menos ser precisos acerca de qué juicios de valor son utilizados en una investigación y por ende de cuáles deben ser usados para ello. La presencia de juicios de valor éticos, que para Carnap implicaría la ausencia de objetividad, es para Rudner parte inalienable de tal objetividad.

Finalmente, Rudner menciona explícitamente a Carnap en su trabajo, al referirse a la distinción carnapiana entre cuestiones internas y cuestiones externas. Mientras las primeras son teóricas y tienen una respuesta con contenido cognitivo, las últimas son prácticas, acerca de las decisiones a llevar a cabo para elegir una estructura de lenguaje en lugar de otra. Estas últimas, nos dice Rudner, son cuestiones de valor, aunque Carnap no haya empleado tal rótulo.

Por lo tanto, nuestra propuesta al respecto va más lejos que la de Rudner acerca de Carnap, porque reconocemos que tales cuestiones de valor operan, según Carnap, también para resolver cuestiones internas como las de aceptación o rechazo de determinadas hipótesis y teorías. Creemos que, también para Carnap, el científico qua científico hace juicios de valor, aunque de un tipo muy particular a discutir a continuación.

\section{Carnap y la preservación de la objetividad científica: juicios relativos y absolutos de valor}

Subyace a toda la propuesta de Carnap sobre la aceptación-rechazo de hipótesis y teorías una muy particular concepción de los juicios de valor.

Carnap distingue entre juicios absolutos o incondicionales y juicios relativos o condicionales de valor. Juicios absolutos de valor son aquellos juicios donde no relativizamos a nada ulterior, distinto o posterior. Por ejemplo, un juicio donde afirmamos que un acto es moralmente bueno o moralmente malo; aquí no estamos relativizando a nada ulterior. Los juicios relativos de valor son aquellos en los que el valor se predica relativizándolo a algo ulterior; por ejemplo, todo juicio instrumental de valor es un juicio relativo de valor; por ejemplo, cuando afirmamos que un acto es bueno porque es instrumental para alcanzar un determinado objetivo.

Nos preguntamos entonces qué tipo de juicios de valor operan en la decisión involucrada en la aceptación-rechazo de hipótesis. Recordemos que, según 
Carnap, las evaluaciones al respecto son elucidables empíricamente por determinadas ciencias (preferentemente por la psicología conductista). Carnap además sostiene que los juicios relativos de valor son empíricos, porque se puede establecer empíricamente si un acto es instrumental para alcanzar un determinado objetivo. Pero los juicios absolutos de valor carecen de contenido informativo empírico. Cuando se los acepta o rechaza, se lo hace, al decir de Carnap, por "razones pragmáticas", en acuerdo con la postura de Dewey al respecto y señalando que en un principio, durante la época del Círculo de Viena, los juicios éticos eran concebidos como solo expresando nuestros estados emotivos. Lo importante es que la ciencia empírica no los abarca y, por lo tanto, carecen de la objetividad propia del conocimiento científico (la única auténticamente disponible para el ser humano).

La pregunta clave ahora es idónde intervienen los juicios de valor, ya sean absolutos o relativos, en las decisiones de los científicos con relación a la adopción-rechazo de hipótesis y teorías?

Carnap jamás contestó explícitamente tal pregunta. Sin embargo, creemos que es posible dar una respuesta sensata, coherente con lo discutido anteriormente. Los juicios relativos de valor intervienen en toda decisión interna, como por ejemplo aquellas para establecer si una hipótesis o teoría es aceptable o no.

Carnap afirma que la elección entre modos de hablar no es una elección entre verdadero o falso, como lo es la decisión acerca de hipótesis o teorías; de ahí que se lo haga pragmáticamente. Involucran, según Carnap, una decisión de nuestra voluntad no reducible a uso de evidencia ni de lógica alguna (con presencia, según Carnap, de "elementos volitivos"). Al adoptar un marco lingüístico, se establecen los candidatos a ser susceptibles de ser verdaderos o falsos, pero cuáles son actualmente verdaderos (falsos) es algo determinado férreamente dentro del marco por el método de aceptación-rechazo debido al testeo empírico. Así como se recomienda que en la decisión para adoptar un determinado marco se sea tolerante, admitiendo la posibilidad de marcos alternativos, en la decisión sobre la aceptación o rechazo de hipótesis o teorías internas al marco, tal tolerancia desaparece y esta debe hacerse en términos de buena lógica inductiva plus valuaciones en términos de preferencia por utilidad, las que, traducidas a la terminología de juicios de valor, significa en términos de juicios relativos de valor.

En dicha propuesta se asume que no hay un marco epistemológico (de segundo nivel) desde el cual sea posible discutir y establecer, en términos de razones epistémicas, la elección entre marcos. No hay tribunal supracientífico alguno desde el cual se juzgue tal elección. Lo único que existe, más allá de la investigación empírica misma, es el análisis lógico de lo que se lleva a cabo dentro de un lenguaje, léase dentro de un determinado marco lingüístico; pero, en base a todo lo discutido, tal análisis lógico, ya sea de lógica deductiva o inductiva, solo es capaz de abordar cuestiones internas a un determinado lenguaje.

Sin embargo, en ambos casos, tanto en la elección de hipótesis dentro de un marco como en la de los marcos, intervienen exclusivamente juicios instrumen- 
tales de valor. Esto quedó claro acerca de las elecciones internas al marco. Sin embargo, debe agregarse que, según Carnap, la elección de marcos se hace en términos de la funcionalidad de la adopción de un determinado marco a la obtención de un determinado objetivo. Por ejemplo, si en la elección de un marco lingüístico para expresar la aritmética el objetivo es postular la existencia entitativa de los números, entonces se ha de adoptar un marco en el que los números pertenezcan al rango de variabilidad de las variables cuantificables. Por supuesto, la discusión racional de los objetivos mismos queda fuera del discurso estrictamente significativo.

Proponemos, pues, que los juicios absolutos de valor no intervienen estrictamente en las cuestiones internas ni en las cuestiones externas (con lo que se salvaguarda la objetividad de la actividad científica), pero parece que juegan un rol más distante.

\section{El valor de la ciencia}

Carnap propone tres tesis que sintetizan su postura acerca del valor de la ciencia: (1) no hay autoridad superior de la cual dependan parasitariamente nuestras concepciones acerca de cosa alguna, (2) el ser humano es el único responsable de lo que pasa, tanto a él mismo como a la sociedad y, en tanto tal, es el único responsable para cambiar o para cambiarla cuando lo considere necesario y (3) la historia misma ha mostrado que la ciencia puede jugar un rol importantísimo "para mejorar la vida" (Carnap 1963, p. 83).

Tal posición es coherente con la declaración del "Círculo de Viena" ([1929] 2002) en la que se enfatizaba que la propuesta general de este merecía el rótulo de "humanismo científico". Todo lo defendido en la vida del positivismo lógico fue funcional, según ellos, a tal humanismo. La eliminación de la metafísica y de la ética como empíricamente significativas, la aspiración a lograr una ciencia unificada involucrando un lenguaje unificado con una lógica común subyacente, la búsqueda de un criterio de significación empírica funcional a la eliminación de la metafísica y la ética de tal ámbito significativo empírico, la obsesión por hallar una interpretación observacional plausible a los términos teóricos, la analiticidad de la lógica y la matemática, son, según Carnap, funcionales a tal programa filosófico. Ello es análogo a afirmar que las célebres dicotomías constitutivas del positivismo lógico, como por ejemplo las dicotomías entre cognitivo-no cognitivo, analítico-sintético y hecho-valor, eran funcionales a tal humanismo científico.

Presenciamos además, y consistentemente con la postura de la llamada "ala izquierda" del Círculo de Viena (a la que pertenecía Carnap junto a Neurath y Frank), una fuerte actitud antifundacionalista: no hay fundamentos últimos fuera de las ciencias desde los cuales justificar afirmaciones acerca de ellas; todo se hace desde dentro de las ciencias: ello se pone de relieve, por ejemplo, en la metáfora del bote de Neurath, así como en las propuestas de Carnap aquí discutidas. No hay en ello atisbo alguno de viciosa circularidad, porque la acepta- 
ción-rechazo de hipótesis de una teoría requerirá del uso de valuaciones bien asentadas por la investigación ya aceptada en otra teoría, y así sucesivamente, "siempre desde dentro del bote" de la investigación científica. No hay nada aceptable desde fuera de tal bote donde asentar con certeza última las decisiones sobre la aceptación-rechazo de hipótesis y teorías.

La ciencia es valiosa para mejorar la vida, es decir, entre otras cosas, para implementar y desarrollar una organización social más justa. Pero Carnap mismo aclara en su Autobiografía (Carnap 1963, p. 83) que todo ello era para él meramente relativo, porque el valor supremo es "una vida libre y fructífera de los individuos" (Carnap 1963, p. 83). Todos los juicios relativos de valor son, en última instancia, instrumentales a un supremo juicio absoluto de valor, estableciendo como valor supremo la plena realización de los individuos en libertad.

En síntesis: los juicios absolutos de valor no intervienen, según Carnap, en las cuestiones internas ni en las cuestiones externas. Pero cuando avanzamos en la consideración de los juicios relativos de valor que intervienen en las cuestiones externas, desembocamos en un último juicio absoluto de valor.

Presenciamos así un esfuerzo notable, por una parte (contra la historia oficial), de aceptar la presencia de cierto tipo de juicios de valor en la actividad científica y, por otra parte, de evitar, hasta el límite de lo posible, la incidencia de ingredientes no cognitivos en la elucidación de tales juicios de valor (todos son en última instancia analizables por alguna ciencia empírica). Parte de la estrategia es la eliminación de la ética del ámbito de lo empíricamente significativo y la no presencia de juicios valorativos éticos en la actividad científica, algo que Putnam hizo bien en criticar.

Presenciamos, además, la incidencia en toda la postura carnapiana de un fuerte ingrediente moderno: la funcionalidad de la ciencia para el progreso individual y social, considerados como objetivos últimos constitutivos de una concepción humanista del conocimiento científico en la que, excepto tal valor último, todos sus ingredientes valorativos son relativos y aclarables empíricamente (en total coherencia con el desplazamiento de la ética de lo empírico-significativo).

Pero, lamentablemente, detectamos por todo ello un límite insuperable de todo empirismo acerca de los valores absolutos: su incapacidad para discutirlos racionalmente. No en vano, Carnap los deja fuera de la actividad científica que, para todo el positivismo, es la actividad racional por excelencia. El reemplazo de la epistemología por la lógica del lenguaje de la ciencia, así como la eliminación de la ética del ámbito de lo empíricamente significativo, coherente con la reducción de la racionalidad a racionalidad lógico-instrumental, determinaron límites infranqueables, imposibilitando una discusión más amplia y rica de la naturaleza de los juicios de valor presentes en la aceptación y rechazo de hipótesis y teorías. 


\section{Bibliografía}

Carnap, R. (1962), Logical Foundations of Probability, Chicago-London: The University of Chicago Press.

Carnap, R. (1963), “Intellectual Autobiography”, en Schilpp (1963), pp. 3-86.

Carnap, R. (1963), “The Philosopher Replies”, en Schilpp (1963), pp. 859-1013.

Frank, Ph. (1954), "The Variety of Reasons for the Acceptance of Scientific Theories", Scientific Monthly 79: 139-145.

Hahn, H., Neurath, O. y Carnap, R., ([1929]2002), "La concepción científica del mundo: el Círculo de Viena”, traducción de Pablo Lorenzano, Redes 18:103-149.

Neurath, O. (1983), Philosophical Papers. 1913-1946, ed. por R. Cohen y M. Neurath, Dordrecht-Boston-Lancaster: D. Reidel Publishing Company.

Putnam, H. (2002), The Collapse of the Fact-Value Dichotomy and Other Essays, Cambridge, MA-London: Harvard University Press.

Rudner, R. (1953), “The Scientist Qua Scientist Makes Value Judgments”, Philosophy of Science 20: 1-6.

Schilpp, P. (ed.) (1963), The Philosophy of Rudolf Carnap, La Salle, Ill.: Open Court. 
